\title{
REAL ORTHOGONAL REPRESENTATIONS OF ALGEBRAIC GROUPS
}

\author{
BY \\ FRANK GROSSHANS
}

\begin{abstract}
The purpose of this paper is to determine explicitly, nondegenerate real symmetric bilinear forms invariant under a real absolutely irreducible representation of a real semisimple algebraic group, $G$. If $G$ is split, we construct an extension $G^{*}$ containing $G$ and those outer automorphisms of $G$ fixing the highest weight of the representation. The representation is then extended to $G^{*}$ and the form is described in terms of the character of this extension. The case of a nonsplit algebraic group is then reduced to the above. The corresponding problem for representations by matrices over the real quaternion division algebra is also considered using similar methods.
\end{abstract}

Introduction. It is a classical result that a real representation of a compact Lie group preserves a definite symmetric bilinear form. For an arbitrary semisimple Lie group, Mal'cev [2] was able to determine those representations which preserve a nondegenerate symmetric (or alternating) bilinear form. More recently, Satake [5] has studied symplectic representations of semisimple algebraic groups.

The purpose of this paper is to determine explicitly, nondegenerate symmetric bilinear forms invariant under an absolutely irreducible representation of a real semisimple algebraic group. This work is a continuation of [1] and gives a further answer to a question raised by Satake [5, p. 251]. For clarity, we shall repeat the problems in which we are interested.

Problem 1. Let $G \subset \mathrm{GL}(n, C)$ be a (connected) semisimple Chevalley group defined over $\boldsymbol{R}$ and let $\rho: G \rightarrow \mathrm{GL}(V)$ be an absolutely irreducible finite-dimensional representation of $G$ defined over $\boldsymbol{R}$ such that ${ }^{t} \rho^{-1} \sim \rho$. Then there is a nondegenerate invariant bilinear form $B$ defined over $\boldsymbol{R}$ on $V$ which we wish to determine. (All of the invariant forms which we shall discuss are unique only up to a scalar multiple.)

To extend our solution to groups not of Chevalley type, we use some results of Satake. Indeed, let $G_{1} \subset \mathrm{GL}\left(n^{\prime}, C\right)$ be a connected semisimple algebraic group defined over $\boldsymbol{R}$ and let $f$ be an isomorphism from $G_{1}$ onto $G$ which is defined over $C$. Then $\rho \circ f$ is an absolutely irreducible representation of $G_{1}$ defined over $C$. We assume that there is a (unique) central simple division algebra $A_{\rho}$ defined over $\boldsymbol{R}$, a finite-dimensional right vector space $V_{1}$ over $A_{\rho}$, and an $\boldsymbol{R}$-homomorphism

Received by the editors October 13, 1970.

AMS 1970 subject classifications. Primary 20G20, 20G05.

Key words and phrases. Algebraic groups, representations, invariant symmetric bilinear forms, invariant quaternion hermitian forms.

Copyright (C) 1971, American Mathematical Society 
$\rho_{1}: G_{1} \rightarrow \mathrm{GL}\left(V_{1} / A_{\rho}\right)$ (the group of all nonsingular $A_{\rho}$-linear endomorphisms of $\left.V_{1}\right)$ such that $(\rho \circ f)(g)=M^{\prime}\left(\rho_{1}(g)\right)$ for all $g$ in $G_{1}$; the mapping $M^{\prime}$ is the unique absolutely irreducible representation of End $\left(V_{1} / A_{\rho}\right)$ (the algebra of all $A_{\rho}$-linear endomorphisms of $V_{1}$ ) onto End $(V)$. (In [5], Satake has shown how such representations arise.) If ${ }^{t} \rho^{-1} \sim \rho$, then $A_{\rho}$ has an involution of the first kind and there is a nondegenerate invariant form defined over $\boldsymbol{R}$ on $V_{1}$.

Problem 2. Assume that $A_{\rho}=\boldsymbol{R}$ and that $B$ is symmetric. Then there exists a nondegenerate invariant symmetric bilinear form $S_{1}$ on $V_{1}$ which we wish to determine.

Problem 3. Assume that $A_{\rho}$ is the quaternion division algebra over $\boldsymbol{R}$ and that $B$ is alternating. Then there is a nondegenerate invariant hermitian form $H$ on $V_{1}$ which we wish to determine.

If $A_{\rho}$ is the quaternion division algebra over $\boldsymbol{R}$ and $B$ is symmetric, then the form $H$ is skew-hermitian and is determined completely by the dimension of the underlying vector space [6, Theorem 2, p. 392]. Hence, we shall not discuss this case.

We begin Part I by proving some basic facts about orthogonal representations of semisimple Chevalley groups. In $\S 1.2$, we associate to a pair $(G, \rho)$ a group $G_{\rho}$, which contains $G$ and those outer automorphisms $\theta$ of $G$ such that $\rho \circ \theta \sim \rho$, and on extension $\rho^{*}$ of $\rho$ to $G_{\rho}$. Our solution to Problem 1 is given in $\S 1.3$ (Theorem 1.6).

We consider Problems 2 and 3 in Part II. Roughly speaking, the invariant bilinear form on a representation of a real semisimple algebraic group $G_{1}$ depends on the difference between $G_{1}$ and its unique compact real form. (A precise formulation is given in Theorems 2.1 and 2.2.) In the Appendix, we discuss a problem in classification theory which arises in Part II.

The author would like to thank Professor I. Satake for his very helpful comments on this paper.

\section{PART I}

1.1. Notation and basic results. In this section, we fix some notation which will be used throughout this paper and then prove some elementary facts about orthogonal representations.

Let $G \subset \mathrm{GL}(n, C)$ be a (connected) semisimple Chevalley group defined over $K$ (a subfield of $C$ ) and let $T$ be a maximal $K$-split torus in $G$. We denote by $\Delta=$ $\left\{\alpha_{1}, \ldots, \alpha_{n}\right\}$ a corresponding fundamental root system and by $W=N(T) / T$ the Weyl group of $T$. We shall always use additive notation on the character module $X(T)$ of $T$.

Let $\rho: G \rightarrow \mathrm{GL}(V)$ be an absolutely irreducible finite-dimensional representation of $G$ defined over $K$ and let $\lambda_{\rho}$ denote the highest weight of $\rho$. We shall write $\Lambda_{\rho}$ for the set of all weights of $\rho$ and for $\lambda$ in $\Lambda_{\rho}$ we define $V^{\lambda}=\{v \in V \mid \rho(t) v=\lambda(t) v$ for all $t$ in $T$ \}. Each subspace $V^{\lambda}$ is defined over $K$.

The absolutely irreducible representation $\rho$ is said to be self-contragredient if $\rho \sim{ }^{t} \rho^{-1}$. In this case, there is a nondegenerate invariant bilinear form $B$ on $V$ 
which is also defined over $K$. In general, such a representation will be denoted by a triple $(V, \rho, B)$ and we shall say that $(V, \rho, B)$ is an orthogonal (or symplectic) representation of $G$ defined over $K$ if $B$ is symmetric (or alternating).

LEMMA 1.1. Let $(V, \rho, B)$ be a self-contragredient representation of $G$ defined over $K$. Then $B \mid V^{\lambda} \times V^{\mu}=0$ unless $\lambda=-\mu$ and $B \mid V^{\lambda} \times V^{-\lambda}$ is nondegenerate.

Proof. Let $x \in V^{\lambda}$ and $y \in V^{\mu}$. For each $t$ in $T, B(x, y)=B(\rho(t) x, \rho(t) y)=$ $(\lambda+\mu)(t) B(x, y)$ and the lemma follows immediately.

If $S$ is a nondegenerate symmetric bilinear form on a vector space $V$ defined over $K$, the Witt index of $S$ over $K$ is the dimension of any maximal totally isotropic subspace of $V$ defined over $K$.

COROllary 1.2. Let $G$ be a semisimple Chevalley group defined over $K$ and let $(V, \rho, S)$ be an orthogonal representation of $G$ defined over $K$ such that 0 is not a weight of $\rho$. Then the Witt index (over $K$ ) of $S$ is $\frac{1}{2} \operatorname{dim} V$.

Proof. The subspaces $W=\sum_{\lambda>0} V^{\lambda}$ and $W^{\prime}=\sum_{\lambda<0} V^{\lambda}$ are totally isotropic (by Lemma 1.1) and are defined over $K$. Since 0 is not a weight of $\rho, \operatorname{dim} W=\frac{1}{2} \operatorname{dim} V$ and the proof is finished.

It can be shown that, for absolutely simple groups, 0 is a weight of every (absolutely irreducible) orthogonal representation of $C_{n}, E_{6}, E_{7}, E_{8}, G_{2}$, and $F_{4}$ [2].

Let $w_{1}=g_{1} T / T$ be the element in $W$ such that $w_{1} \Delta=-\Delta$. It is well known that $\rho$ is self-contragredient if and only if $w_{1} \lambda_{\rho}=-\lambda_{\rho}$. Furthermore, $g_{1}^{2}=t_{1} \in T$ and if $\lambda$ is any character of $T$ such that $w_{1} \lambda=-\lambda$, then $\lambda\left(t_{1}\right)^{2}=1$ since $g_{1}$ and $t_{1}$ commute. (Of course, the element $t_{1}$ depends on our choice of $g_{1}$. However, in what follows we do not require that $t_{1}$ be uniquely determined. Indeed, if $w_{1} \lambda=-\lambda$, it is easy to show that $\lambda\left(g_{1}^{2}\right)$ does not depend on the choice of $g_{1}$.)

LEMMA 1.3. Let $(V, \rho, B)$ be a self-contragredient absolutely irreducible representation of $G$ with highest weight $\lambda_{\rho}$. Then $\rho$ is orthogonal if $\lambda_{\rho}\left(t_{1}\right)=1$ and is symplectic if $\lambda_{\rho}\left(t_{1}\right)=-1$. If 0 is a weight of $\rho$, then $\rho$ is orthogonal.

Proof. Let $x$ be a nonzero highest weight vector in $V$. Then $\rho\left(g_{1}\right) x$ is a lowest weight vector in $V$ and, by Lemma 1.1,

$$
0 \neq B\left(x, \rho\left(g_{1}\right) x\right)=B\left(\rho\left(g_{1}\right) x, \rho\left(t_{1}\right) x\right)=\lambda_{\rho}\left(t_{1}\right) B\left(\rho\left(g_{1}\right) x, x\right)
$$

and the first part of the lemma is proved.

It is not hard to show that $g_{1}$ may be chosen so that $g_{1}^{2}$ is in $Z(G)$, the center of $G$. Hence, if 0 is a weight of $\rho$, then $\rho(Z(G))=\{1\}$ and the lemma is proved.

REMARK. A stronger result than Lemma 1.3 is known. Indeed, for a selfcontragredient representation $(V, \rho, B)$ it can be shown that $2 \lambda_{\rho}=\sum_{i=1} m_{i} \alpha_{i}$ where each $m_{i}$ is an integer. Then, writing ${ }^{t} B=e B$, one has $e=(-1)^{m}$ where $m=\sum_{i=1}^{n} m_{i}$.

1.2. The group $G_{\rho}$. In this section, we extend some results of [1]. Let $G, T$, and $\Delta$ be as before and, furthermore, assume that $G$ is simply connected. The auto- 
morphism group of $G$ is a semidirect product of a finite group $\Theta$ and the inner automorphisms of $G$. Furthermore, $\Theta$ can be chosen so that each $\theta$ in $\Theta$ is defined over $K, \theta(T)=T$, and $\theta(\Delta)=\Delta$.

REMARK. It is possible to give an explicit form for elements of $\Theta$. Indeed, let (SS be the Lie algebra of $G$ and let $\mathfrak{B S}=\mathfrak{h}+\sum\left\{E_{\alpha}\right\}$ where $\mathfrak{h}$ is the Lie algebra of $T$ and the $\left\{E_{\alpha}\right\}$ are one-dimensional root spaces. We assume that the $E_{\alpha}$ form a "Chevalley basis" of (S). By this we mean that $\left[E_{\alpha}, E_{\beta}\right]=N_{\alpha, \beta} E_{\alpha+\beta}$ where $\alpha, \beta$ and $\alpha+\beta$ are roots with respect to $T$ and $N_{\alpha, \beta}$ is an integer; furthermore, $\left[E_{\alpha}, E_{-\alpha}\right]$ $=H_{\alpha} \in \mathfrak{h}$ where $\left(H_{\alpha}, H\right)=\alpha(H)$ for all $H \in \mathfrak{h}$. (We denote by $($,$) the Killing form$ on $\mathfrak{h}$.) If $\Phi$ is an automorphism of $G$, we shall denote also by $\Phi$ the induced automorphism of (S). If $\Phi(T)=T$, then $\Phi\left(E_{\alpha}\right)=\mathscr{N}_{\alpha} E_{\Phi(\alpha)}$. We define $\Theta$ to be the group of automorphisms $\theta$ of $G$ such that $\theta(T)=T, \theta(\Delta)=\Delta$, and each $\mathscr{N}_{\alpha}=1$ for $\alpha \in \Delta$. Since $T$ splits over $K$, each $\theta$ is defined over $K$.

Let $\rho: G \rightarrow \mathrm{GL}(V)$ be an absolutely irreducible representation of $G$ defined over $K$ and let $\Theta_{\rho}$ be the subgroup of $\Theta$ consisting of those $\theta$ such that $\rho \circ \theta \sim \rho$. We define an algebraic group $G_{\rho}$ to be $G \cdot \Theta_{\rho}$, the semidirect product of $G$ and $\Theta_{\rho}$ where group multiplication is given by

$$
(g, \theta)\left(g_{1}, \theta_{1}\right)=\left(g \theta\left(g_{1}\right), \theta \theta_{1}\right)
$$

We shall consider $G$ (resp. $\Theta_{\rho}$ ) as a subgroup of $G_{\rho}$ identifying $g$ and $(g, 1)$ (resp. $\theta$ and $(1, \theta)$ ). Our first aim is to extend $\rho$ to $G_{\rho}$ preserving certain properties of $\rho$.

Lemma 1.4. Let $G, \rho$, and $G_{\rho}$ be as above and let $x$ be a nonzero K-rational highest weight vector in $V$.

(i) There exists a unique representation $\rho^{*}: G_{\rho} \rightarrow \mathrm{GL}(V)$ defined over $K$ satisfying $\rho^{*}(g)=\rho(g)$ for each $g$ in $G$ and $\rho^{*}(\theta) x=x$ for each $\theta$ in $\Theta_{\rho}$.

(ii) If $\rho$ is self-contragredient and preserves the nondegenerate bilinear form $B$, then $B$ is also invariant with respect to the representation $\rho^{*}$.

Proof. We denote by $\lambda$ the highest weight of $\rho$. Let $\theta$ be an element in $\Theta_{\rho}$. Since $\rho$ and $\rho \circ \theta$ are both defined over $K$, there is an element $A$ in $\operatorname{GL}(V, K)$ such that $\rho(\theta(g))=A \rho(g) A^{-1}$ for each $g$ in $G$. Since $\theta \lambda=\lambda$, there is an $a$ in $K^{*}$ such that $A x=a x$. We put $\rho^{*}(\theta)=a^{-1} A$. Next we show that $\rho^{*}\left(\theta \theta_{1}\right)=\rho^{*}(\theta) \rho^{*}\left(\theta_{1}\right)$ for each $\theta$ and $\theta_{1}$ in $\Theta_{\rho}$. By Schur's lemma, there is a $c$ in $K^{*}$ such that $\rho^{*}\left(\theta \theta_{1}\right)$ $=c \rho^{*}(\theta) \rho^{*}\left(\theta_{1}\right)$. Applying both sides to $x$, we see that $c=1$. To complete (i), we define $\rho^{*}(g, \theta)=\rho(g) \rho^{*}(\theta)$.

In order to prove (ii), we introduce the group $G^{\theta}=\{g \in G \mid \theta(g)=g\}$. There are regular elements of $G$ in $T \cap G^{\theta}$. (For let the order of $\theta$ be $n$ and let $t$ be an element in $T$ so that, for each positive root $\alpha$ of $T,\left(\alpha+\theta \alpha+\cdots+\theta^{n-1} \alpha\right)(t) \neq 1$. Then $t \theta(t) \cdots \theta^{n-1}(t)$ is regular in $G$ and is in $T \cap G^{\theta}$.) Let $t$ be such an element and let $S$ be its centralizer in $G^{\theta}$. Then $S \subset T$ and $T=Z_{G}(S)$ (the centralizer of $S$ in $G$ ). Furthermore, we see that each nonzero root of $T$ gives rise to a nonzero root of $S$. 
Let $X_{1}=\{\chi \in X(T) \mid \chi(s)=1$ for all $s$ in $S\}$. Then $X(S)$ may be identified with $X(T) / X_{1}$. Let " $>$ " be an order on $X(T)$ compatible with $X_{1}$. Such an order determines uniquely orders on $X_{1}$ and $X(T) / X_{1}$ so that the following property holds: if $\chi$ is not in $X_{1}$, then $\chi>0$ if and only if $\chi+X_{1}>0$. We denote by $\mathfrak{r}_{T}$ and $\mathfrak{r}_{S}$ the positive roots of $X(T)$ and $X(S)$ relative to this order. Let $g$ be an element in $G^{\theta}$ normalizing $S$ so that $I_{g}$ maps $\mathfrak{r}_{S}$ to $-\mathfrak{r}_{S}$. Then $g$ normalizes $T=Z_{G}(S)$ and, clearly, $I_{g}$ maps $\mathfrak{r}_{T}$ to $-\mathfrak{r}_{T}$.

Now, by Schur's lemma, there is an element $\lambda_{\theta}$ in $K^{*}$ such that ${ }^{t} \rho^{*}(\theta) B \rho^{*}(\theta)=\lambda_{\theta} B$. But we have that

$$
\begin{aligned}
0 & \neq B(x, \rho(g) x)=B\left(\rho^{*}(\theta)^{-1} x, \rho(g) \rho^{*}(\theta)^{-1} x\right) \\
& =B\left(\rho^{*}(\theta)^{-1} x, \rho^{*}(\theta)^{-1} \rho(\theta(g)) x\right)=\lambda_{\theta}^{-1} B(x, \rho(g) x)
\end{aligned}
$$

and so $\lambda_{\theta}=1$. This completes the proof of the lemma.

1.3. Problem 1. In this section, we shall give a solution to Problem 1. Before doing so, however, we shall state a well-known result of fundamental importance in this paper. In this theorem, we do not require $G$ to be of Chevalley type [3].

THEOREM 1.5. Let $G$ be a connected semisimple linear algebraic group defined over $R$. Then

(a) in a suitable R-rational basis of the underlying vector space, ${ }^{t} g \in G$ for all $g \in G$;

(b) if $\rho: G \rightarrow \mathrm{GL}(V)$ is a representation of $G$ defined over $R$ and if ${ }^{t} g \in G$ for all $g \in G$, then there exists an $R$-rational basis of $V$ such that $\rho\left({ }^{t} g\right)={ }^{t} \rho(g)$ (in this basis) for all $g$ in $G$.

We recall that a real nondegenerate symmetric bilinear form $S$ on a real vector space $V$ is determined by the following two invariants: $\operatorname{dim} V$ and the signature of $S$, $\operatorname{sgn} S$, which is the number of $(+1)$ 's minus the number of $(-1)$ 's in any diagonal form of $S$. Furthermore, if $S^{2}=1$, it follows that $\operatorname{sgn} S=\operatorname{tr}(S)$.

In stating the next result, we shall use the notation introduced in $\$ 1.2$ concerning the finite group $\Theta$ and the representation $\rho^{*}$ of the group $G_{\rho}$. For $g \in G$, we denote by $I_{g}$ the inner automorphism of $G$ induced by $g$, i.e., $I_{g}(h)=g h g^{-1}$ for all $h$ in $G$.

THEOREM 1.6. Let $G \subset \mathrm{GL}(n, C)$ be a (connected) semisimple linear Chevalley group defined over $\boldsymbol{R}$ such that ${ }^{t} g \in G$ for all $g \in G$. Denote by $\psi$ the automorphism of $G$ given by $g \rightarrow{ }^{t} g^{-1}$ and let $\psi=\theta_{1} \circ I_{g_{1}}$ where $\theta_{1} \in \Theta$ and $g_{1}$ is an R-rational element of $G$. If $(V, \rho, B)$ is an absolutely irreducible self-contragredient representation of $G$ defined over $\boldsymbol{R}$, then there is an $\boldsymbol{R}$-rational basis of $V$ such that (in this basis) $B=\rho^{*}\left(\theta_{1} g_{1}\right)$ up to a scalar multiple. Furthermore, if $B$ is symmetric, $\operatorname{sgn} B$ $= \pm \chi^{*}\left(\theta_{1} g_{1}\right)$ where $\chi^{*}$ is the character of $\rho^{*}: G_{\rho} \rightarrow \mathrm{GL}(V)$.

We note the assumption ${ }^{t} g \in G$ for all $g \in G$ is harmless by Theorem 1.5(a). Furthermore, since $\rho$ is self-contragredient, $\rho \circ \psi \sim \rho$ and so $\rho \circ \theta_{1} \sim \rho$, i.e., $\theta_{1}$ is in 
the group $\Theta_{\rho}$. We may assume that the maximal $\boldsymbol{R}$-split torus $T$ of $G$ has been chosen so that $\psi(T)=T$. Hence, $\psi(\Delta)=-\Delta$ and it follows that $g_{1} \in N(T)$ and $g_{1} T / T=w_{1}: \Delta \rightarrow-\Delta$.

Proof of Theorem 1.6. By Theorem 1.5(b), there is an $R$-rational basis of $V$ such that (relative to this basis) $\rho\left({ }^{t} g\right)={ }^{t} \rho(g)$ for all $g$ in $G$. Hence, by Schur's lemma, in this basis $B=e \rho^{*}\left(\theta_{1} g_{1}\right)$ where $e \in R^{*}$. In particular, if $B$ is symmetric, then $\operatorname{sgn} B= \pm \operatorname{sgn} \rho^{*}\left(\theta_{1} g_{1}\right)$. Hence, to complete the proof, we only have to show that $\rho^{*}\left(\theta_{1} g_{1}\right)^{2}=1$. But since $\psi^{2}=1$, it follows that $\theta_{1}^{2}=1$ and $\theta_{1}\left(g_{1}\right) g_{1} \in Z(G)$. Therefore, $\rho^{*}\left(\theta_{1} g_{1}\right)^{2}=\rho\left(\theta_{1}\left(g_{1}\right) g_{1}\right)= \pm 1$. However, $\rho^{*}\left(\theta_{1} g_{1}\right)$ is a real symmetric matrix and so all of its characteristic roots are real. This proves the theorem.

\section{PART II}

2.1. Problem 2. We shall continue to use the notation of Part I although $K$ will now always be $\boldsymbol{R}$. Complex conjugation will be denoted by $\sigma$ and if a function $f$ has an inverse, we shall write $f^{-\sigma}$ instead of $\left(f^{-1}\right)^{\sigma}$. We shall assume that $G$ is a simply-connected linear semisimple Chevalley group defined over $\boldsymbol{R}$ such that ${ }^{t} g \in G$ for all $g \in G$. The automorphism $g \rightarrow{ }^{t} g^{-1}$ of $G$ will again be denoted by $\psi$.

Let $G_{1}$ be an algebraic group defined over $\boldsymbol{R}$ which is isomorphic to $G$ over $\boldsymbol{C}$ and let $f: G_{1} \rightarrow G$ be an isomorphism.

(1) It is a well-known result of Chevalley that $f$ can be chosen so that $f^{\sigma} \circ f^{-1}$ $=\theta_{0} \circ I_{g_{0}}$ where $\theta_{0} \in \Theta$ and $g_{0} \in N(T)$. Then it follows that $\theta_{0}^{2}=1$ and $\theta_{0}\left(g_{0}\right) g_{0}^{\sigma}$ is in $Z(G)$. In the Appendix, we shall prove the following refinement of this result: The isomorphism $f$ can be chosen so that if $\alpha=\theta_{0} \circ I_{g_{0}}$ then $\alpha^{\sigma}=\alpha$ (i.e., there is an element $z_{0} \in Z(G)$ such that $\left.g_{0}^{\sigma}=g_{0} z_{0}\right)$ and $(\psi \circ \alpha)^{2}=1$. We shall now fix throughout Part II such an isomorphism and shall denote $\psi \circ \alpha$ by $\beta$.

(2) Let $(V, \rho, B)$ be an absolutely irreducible self-contragredient representation of $G$ defined over $\boldsymbol{R}$. Then $\rho\left(\theta_{0}\left(g_{0}\right) g_{0}^{\sigma}\right)$ is \pm 1 and determines the division algebra $A_{\rho}$; indeed, $A_{\rho}=R$ if and only if $\rho\left(\theta_{0}\left(g_{0}\right) g_{0}^{\sigma}\right)=1[5$, p. 227].

(3) Let $\rho_{1}: G_{1} \rightarrow \mathrm{GL}\left(V_{1}\right)$ be an absolutely irreducible representation of $G_{1}$ defined over $\boldsymbol{R}$ and let $\rho: G \rightarrow \mathrm{GL}(V)$ be an absolutely irreducible representation of $G$ defined over $R$ such that $\rho \sim \rho_{1} \circ f^{-1}$. Since $\rho_{1}^{\sigma}=\rho_{1}$, it follows that $\rho \circ \theta_{0} \sim \rho$ and, hence, $\theta_{0}$ is in the group $\Theta_{\rho}$.

The purpose of this section is to prove the next result. In stating it, we shall use the notation introduced in (1) and (2) above.

THEOREM 2.1. Let $(V, \rho, S)$ be an (absolutely irreducible) orthogonal representation of $G$ defined over $\boldsymbol{R}$ such that $\rho \circ \theta_{0} \sim \rho$ and $\rho\left(\theta_{0}\left(g_{0}\right) g_{0}^{\sigma}\right)=1$. Then there exists an orthogonal representation $\left(V_{1}, \rho_{1}, S_{1}\right)$ of $G_{1}$ defined over $\boldsymbol{R}$ such that $\rho_{1} \sim \rho \circ f$.

(i) If $\rho\left(z_{0}\right)=1$, then in a suitable $R$-rational basis of $V_{1}$, the form $S_{1}=\rho^{*}(\beta)$ up to a scalar multiple. Furthermore, $\operatorname{dim} V=\operatorname{dim} V_{1}$ and $\operatorname{sgn} S_{1}= \pm \operatorname{sgn}\left({ }^{t} \rho^{*}\left(\theta_{0} g_{0}\right) S\right)$ $= \pm \chi^{*}(\beta)$ where $\chi^{*}$ is the character of $\rho^{*}: G_{\rho} \rightarrow O(V, S)$.

(ii) If $\rho\left(z_{0}\right)=-1$, then $\operatorname{dim} V=\operatorname{dim} V_{1} \equiv 0(\bmod 2)$ and both of the forms $S$ and $S_{1}$ have maximal Witt index. 
Notation. As before, if $\beta=\theta \circ I_{g}$ we shall write $\rho^{*}(\beta)$ for $\rho^{*}(\theta \circ g)$.

Proof. We set $P=\rho^{*}\left(\theta_{0} g_{0}\right)$; by our assumptions, $(\rho \circ f)^{\sigma}=P(\rho \circ f) P^{-1}$ where $P \in O(V, S)$ (by Lemma 1.4) and $P P^{\sigma}=1$.

(i) If $\rho\left(z_{0}\right)=1$, then $P=P^{\sigma}$ and so $P^{2}=1$. The +1 and -1 eigenspaces of $P$ are perpendicular and so we may find an orthogonal basis $\left\{v_{1}, \ldots, v_{n}\right\}$ of $V$ composed of characteristic vectors of $P$ and defined over $\boldsymbol{R}$. Furthermore, we may assume that $S\left(v_{i}, v_{i}\right)= \pm 1$ for $i=1, \ldots, n$. Let $D$ be the linear transformation of $V$ which is expressed (in the basis $\left\{v_{i}\right\}$ ) by the following diagonal matrix: $D v_{i}=v_{i}$ if $P v_{i}=v_{i}$ of $D v_{i}=\sqrt{ }(-1) v_{i}$ if $P v_{i}=-v_{i}$. Then $P=D^{-\sigma} D$ and, since $D^{-\sigma}=D$, we see that $P=D^{2}$. Using these facts, it can be shown that the representation $\rho_{1}=$ $D(\rho \circ f) D^{-1}$ and the form $S_{1}={ }^{t} D^{-1} S D^{-1}$ have the desired properties for $\left(V_{1}, \rho_{1}, S_{1}\right)$. Furthermore, $\operatorname{sgn} S_{1}=\operatorname{sgn}^{t} D^{\sigma} S D^{\sigma}=\operatorname{sgn}{ }^{t} P S$.

As we saw in (1), $\beta=\psi \circ \theta_{0} \circ I_{g_{0}}$. By Theorem 1.6, $S=\rho^{*}(\psi)$ is a suitable $\boldsymbol{R}$ rational basis of $V$ and $S^{2}=1$. Hence, in this basis, $\operatorname{sgn} S_{1}=\operatorname{sgn} \rho^{*}(\beta)$. We show that $\rho^{*}(\beta)^{2}=1$ just as in the proof of Theorem 1.6. Hence, $\operatorname{sgn} S_{1}= \pm \chi^{*}(\beta)$ and the proof of (i) is complete.

(ii) If $\rho\left(z_{0}\right)=-1$, then $P=-P^{\sigma}$ and so $P^{2}=-1$. We put

$$
V^{+}=\{v \in V \mid P v=\sqrt{ }(-1) v\}
$$

and $V^{-}=\{v \in V \mid P v=-\sqrt{ }(-1) v\}$. The subspaces $V^{+}$and $V^{-}$are defined over $\boldsymbol{R}$, $S \mid V^{+} \times V^{+}=0$ and $S \mid V^{-} \times V^{-}=0$. Hence, if $m=\operatorname{dim} V^{+}$, then $\operatorname{dim} V=2 m$. We choose $\boldsymbol{R}$-rational bases $\left\{v_{1}, \ldots, v_{m}\right\}$ of $V^{+}$and $\left\{v_{m+1}, \ldots, v_{2 m}\right\}$ of $V^{-}$so that $S\left(v_{i}, v_{m+j}\right)=\delta_{i j}$. Let $\zeta=\exp \left(\frac{1}{4} \pi \sqrt{ }(-1)\right)$ and let $D$ be the linear transformation of $V$ which is expressed (in the basis $\left\{v_{i}\right\}$ ) by the following diagonal matrix: $D v_{i}=\zeta v_{i}$ for $i=1, \ldots, m$ and $D v_{i}=\zeta^{3} v_{i}$ for $i=m+1, \ldots, 2 m$. Then $P=D^{-\sigma} D$ and since $D^{-\sigma}=D$, we see that $P-D^{2}$. Using these facts, it can be shown that the representation $\rho_{1}=D(\rho \circ f) D^{-1}$ and the form $S_{1}={ }^{t} D^{-1} S D^{-1}$ have the desired properties for $\left(V_{1}, \rho_{1}, S_{1}\right)$. Furthermore, it is easy to show that $S_{1}$ has maximal Witt index. This completes the proof of the theorem.

Remark. Our earlier result, Theorem 1.6, is contained in the statement of Theorem 2.1 if we put $G=G_{1}, f=1_{G}$. Furthermore, the unique compact real form $G_{u}$ of $G$ is an $R$-form of $G$ which corresponds to the 1-cocycle $\left\{\Phi_{\tau}\right\}$ where $\Phi_{1}=1$ and $\Phi_{\sigma}=\psi$ i.e., there is an isomorphism $f: G_{u} \rightarrow G$ defined over $C$ such that $f^{\sigma} \circ f^{-1}=\psi$. Hence, from Theorem 2.1 setting $G_{1}=G_{u}$, we obtain the classical result that the form $S_{1}$ is definite.

2.2. Problem 2. Throughout this section, we shall denote by $\mathfrak{t}$ the quaternion division algebra over $\boldsymbol{R}$. We begin by recalling some results about $\mathfrak{f}$. The algebra $\mathfrak{f}$ has a basis $\left(1, x_{1}, x_{2}, x_{1}, x_{2}\right)$ over $R$ such that $x_{1}^{2}=-1, x_{2}^{2}=-1$, and $x_{1} x_{2}=-x_{2} x_{1}$. An isomorphism $M: \mathfrak{t} \rightarrow \mathfrak{l}_{C}$ is given by

$$
M\left(Y_{0}+Y_{1} x_{1}+Y_{2} x_{2}+Y_{3} x_{1} x_{2}\right)=\left(\begin{array}{cc}
Y_{0}+Y_{1} \sqrt{ }(-1) & -\left(Y_{2}+Y_{3} \sqrt{ }(-1)\right) \\
Y_{2}-Y_{3} \sqrt{ }(-1) & Y_{0}-Y_{1} \sqrt{ }(-1)
\end{array}\right) .
$$


The map $M$ is defined over $C$ and $M^{\sigma}(x)=M\left(n_{\sigma}^{-1} x n_{\sigma}\right)$ for all $x \in \mathfrak{f}$ where $n_{\sigma}=x_{2}$. The function $x \rightarrow \bar{x}$ of $\mathfrak{t}$ to $\mathfrak{t}$ given by $x=Y_{0}+Y_{1} x_{1}+Y_{2} x_{2}+Y_{3} x_{1} x_{2} \rightarrow \bar{x}=$ $Y_{0}-Y_{1} x_{1}-Y_{2} x_{2}-Y_{3} x_{1} x_{2}$ is an involution of the first kind on $\mathfrak{f}$. Furthermore, $M(\bar{x})=J^{-1}{ }^{t} M(x) J$ where

$$
J=\left(\begin{array}{rr}
0 & -1 \\
1 & 0
\end{array}\right)
$$

Let $V_{1}$ be a finite-dimensional right vector space over $\mathfrak{f}$ and let $H$ be a hermitian form of $V_{1}$ (with respect to the involution above). We may define sgn $H$ as we did for symmetric forms. Then the two invariants $\operatorname{dim} V_{1}$ (over $\mathfrak{f}$ ) and $\operatorname{sgn} H$ determine $H$. In stating the next result, we again shall use the notation of (1) and (2) in $\S 2.1$.

THEOREM 2.2. Let $(V, \rho, A)$ be an absolutely irreducible symplectic representation of $G$ defined over $\boldsymbol{R}$ such that $\rho \circ \theta_{0} \sim \rho$ and $\rho\left(\theta_{0}\left(g_{0}\right) g_{0}^{\sigma}\right)=-1$. Then there exists a hermitian representation $\left(V_{1} \mid \mathfrak{k}, \rho_{1}, H\right)$ of $G_{1}$ defined over $\boldsymbol{R}$ such that $(\rho \circ f)(g)$ $=M^{\prime}\left(\rho_{1}(g)\right)$ for all $g$ in $G_{1}$ where $M^{\prime}:$ End $\left(V_{1} / \mathfrak{t}\right) \rightarrow$ End $(V)$ is the absolutely irreducible isomorphism. Furthermore, $\operatorname{dim} V_{1}($ over $\mathfrak{t})=\frac{1}{2} \operatorname{dim} V$.

(i) If $\rho\left(z_{0}\right)=1$, then $\operatorname{sgn} H= \pm \frac{1}{2} \chi^{*}(\beta)$ where $\chi^{*}$ is the character of

$$
\rho^{*}: G_{\rho} \rightarrow \operatorname{Sp}(V, A) \text {. }
$$

(ii) If $\rho\left(z_{0}\right)=-1$, then $\operatorname{sgn} H= \pm \frac{1}{2} \sqrt{ }(-1) \chi^{*}(\beta)$ where $\chi^{*}$ is the character of $\rho^{*}: G_{\rho} \rightarrow \operatorname{Sp}(V, A)$.

Satake has shown the existence of $\left(V_{1} / \mathfrak{k}, \rho_{1}, H\right)[5$, p. 230]. We shall outline here some of the pertinent facts about that existence argument. If $e_{i j}(i, j=1, \ldots, 4)$ are matrix units in $\mathfrak{f}$, then considering $V_{1} e_{11}$ as a vector space over $C$, there is a unique (up to a scalar multiple) $C$-isomorphism $f_{1}: V \rightarrow V_{1} e_{11}$ such that

$$
R_{n_{\sigma}}=a_{\sigma} f_{1} \circ P^{-1} \circ f_{1}^{-\sigma}
$$

where $R_{n_{o}}: V_{1} e_{22} \rightarrow V_{1} e_{11}$ is given by $R_{n_{\sigma}}(v)=v n_{\sigma}$ for $v$ in $V_{1} e_{22}$. The map $P$ is $\rho^{*}\left(\theta_{0} g_{0}\right)$ and $a_{\sigma}$ is in $C$.

Define $B_{11}$ on $V_{1} e_{11}$ so that $f_{1}$ is an isomorphism of $(V, A)$ with $\left(V_{1}, B_{11}\right)$ and set $B_{i j}(v, w)=B_{11}\left(v e_{i 1}, w e_{j 1}\right)$ for all $v, w$ in $V_{1}$ and $i, j=1,2,3,4$. The form $H$ is defined by the formula

$$
J M(H(v, w))=\left(B_{i j}(v, w)\right)
$$

is hermitian if $B_{11}$ is alternating.

Proof of Theorem 2.2. We set $P=\rho^{*}\left(\theta_{0} g_{0}\right)$; by our assumptions, $(\rho \circ f)^{\sigma}=$ $P(\rho \circ f) P^{-1}$ where $P \in \operatorname{Sp}(V, A)$ (by Lemma 1.4) and $P P^{\sigma}=-1$.

(i) If $\rho\left(z_{0}\right)=1$, then $P=P_{\sigma}$ and $P^{2}=-1$.

We define subspaces

$$
V^{+}=\{v \in V \mid P v=\sqrt{ }(-1) v\} \text { and } V^{-}=\{v \in V \mid P v=-\sqrt{ }(-1) v\} .
$$


Since $P \in \operatorname{Sp}(V, A)$, it follows that $A\left|V^{+} \times V^{+}=A\right| V^{-} \times V^{-}=0$ and so $\operatorname{dim} V^{+}$ $=\operatorname{dim} V^{-}$. (As a matter of fact, $\left(V^{+}\right)^{\sigma}=V^{-}$.) Let $x_{1}, \ldots, x_{m}$ be an orthogonal $\boldsymbol{R}$-rational basis of $V_{1}$ such that $H\left(x_{i}, x_{i}\right)=1$ or -1 for all $i$. If $v_{1}, \ldots, v_{m}$ is a basis of $V^{+}$, then $v_{1}^{\sigma}, \ldots, v_{m}^{\sigma}$ is a basis of $V^{-}$and we may define $f_{1}: V \rightarrow V_{1} e_{11}$ by $f_{1}\left(v_{i}\right)=x_{i} e_{11}$ and $f_{1}\left(v_{i}^{\sigma}\right)=-\sqrt{ }(-1) x_{i} e_{21}$. Then $R_{n_{\sigma}}=(-1) f_{1} \circ P \circ f_{1}^{-\sigma}$ and so $f_{1}$ is as in (1) with $a_{\sigma}=-1$.

We define a hermitian form $H^{+}$on $V^{+}$by $H^{+}(x, y)=\sqrt{ }(-1) A\left(x, y^{\sigma}\right)$. Then $\operatorname{sgn} H=\operatorname{sgn}\left(-H^{+}\right)$. In the real basis $\left\{w_{1}, \ldots, w_{2 m}\right\}$ of $V$ given by $w_{i}=v_{i}+v_{i}^{\sigma}$, $w_{m+i}=\sqrt{ }(-1)\left(v_{1}-v_{i}^{\sigma}\right)$ for $i=1, \ldots, m$, the matrix of $P$ is

$$
\left(\begin{array}{cc}
0 & -1_{m} \\
1_{m} & 0
\end{array}\right)
$$

In this basis, the matrix of $A$ is

$$
\left(\begin{array}{rr}
0 & S \\
-S & 0
\end{array}\right)
$$

where $S$ is a real symmetric $m \times m$ matrix. Finally, since

$$
A\left(w_{i}, w_{m+j}\right)=-\left[H^{+}\left(v_{i}, v_{j}\right)+H^{+}\left(v_{i}, v_{j}\right)^{\sigma}\right],
$$

it follows that $\operatorname{sgn}\left(-H^{+}\right)=+\operatorname{sgn} S=\frac{1}{2} \operatorname{sgn}\left(-{ }^{t} P A\right)$.

We conclude this proof by a familiar argument. Indeed, by Theorem 1.6, there is an $\boldsymbol{R}$-rational basis of $V$ such that (in this basis) $A=\rho^{*}(\psi)$ up to a scalar multiple. Hence, in this basis, $-{ }^{t} P A=\rho^{*}(\beta)$ up to a scalar multiple and, as before, $\rho^{*}(\beta)^{2}=1$. This completes the proof of (i).

(ii) If $\rho\left(z_{0}\right)=-1$, then $P=-P^{\sigma}$ and so $P^{2}=1$. We define subspaces

$$
V^{+}=\{v \in V \mid P v=v\} \text { and } V^{-}=\{v \in V \mid P v=-v\} \text {. }
$$

Since $P \in \operatorname{Sp}(V, A)$, it follows that $A \mid V^{+} \times V^{-}=0$. Furthermore, $\left(V^{+}\right)^{\sigma}=V^{-}$and, hence, $\operatorname{dim} V^{+}=\operatorname{dim} V^{-}$. Let $x_{1}, \ldots, x_{m}$ be an orthogonal $R$-rational basis of $V_{1}$ such that $H\left(x_{i}, x_{i}\right)=1$ or -1 for all $i$. If $v_{1}, \ldots, v_{m}$ is a basis of $V^{+}$, then $v_{1}^{\sigma}, \ldots, v_{m}^{\sigma}$ is a basis of $V^{-}$and we may define $f_{1}: V \rightarrow V_{1} e_{11}$ by $f_{1}\left(v_{i}\right)=x_{i} e_{11}$ and $f_{1}\left(v_{i}^{\sigma}\right)$ $=-\sqrt{ }(-1) x_{i} e_{21}$. Then $R_{n_{\sigma}}=(-\sqrt{ }(-1)) f_{1} \circ P \circ f_{1}^{-\sigma}$ and so $f_{1}$ is as in (1) with $a_{\sigma}=-\sqrt{ }(-1)$.

We define a hermitian form $H^{+}$on $V^{+}$by $H^{+}(x, y)=\sqrt{ }(-1) A\left(x, y^{\sigma}\right)$. Then $\operatorname{sgn} H=\operatorname{sgn}\left(-H^{+}\right)$. In the real basis $\left\{w_{1}, \ldots, w_{2 m}\right\}$ of $V$ given by $w_{i}=v_{i}+v_{i}^{\sigma}$, $w_{m+1}=\sqrt{ }(-1)\left(v_{i}-v_{i}^{\sigma}\right)$ for $i=1, \ldots, m$, the matrix of $P$ is

$$
\left(\begin{array}{cc}
0 & +\sqrt{ }(-1) \\
-\sqrt{ }(-1) & 0
\end{array}\right) .
$$

In this basis, the matrix of $A$ is

$$
\left(\begin{array}{rr}
0 & S \\
-S & 0
\end{array}\right)
$$


where $S$ is a real symmetric $m \times m$ matrix. Finally, since

$$
A\left(w_{i}, w_{j}\right)=-\left[H^{+}\left(v_{i}, v_{j}\right)+H^{+}\left(v_{i}, v_{j}\right)^{\sigma}\right],
$$

it follows that $\operatorname{sgn}\left(-H^{+}\right)=\operatorname{sgn} S=\frac{1}{2} \operatorname{sgn}\left(-\sqrt{ }(-1)^{t} P A\right)$.

As before, ${ }^{t} P A=\rho^{*}(\beta)$ up to a scalar multiple and $\rho^{*}(\beta)^{2}=-1$. Hence, $\operatorname{sgn} H$ $=\operatorname{sgn} S= \pm \frac{1}{2} \sqrt{ }(-1) \chi^{*}(\beta)$. This completes the proof of the theorem.

REMARK. Taking $G_{1}=G_{u}$, we see that the corresponding hermitian form is definite.

Appendix. The purpose of this appendix is to prove the refinement of the fundamental theorem of Chevalley given in statement (1), \$2.1. We shall rely mainly on some results in the classification theory of real simple Lie algebras which can be found in [4].

Let $G$ be a simply-connected absolutely simple linear Chevalley group defined over $\boldsymbol{R}$ such that ${ }^{t} g^{-1} \in G$ for each $g \in G$. We shall denote by $\psi$ the automorphism $g \rightarrow{ }^{t} g^{-1}$ of $G$ and shall fix a maximal $\boldsymbol{R}$-split torus $T$ of $G$ such that $\psi(T)=T$. The unique compact real form $G_{u}$ of $G$ is an $R$-form of $G$ which corresponds to the 1-cocycle $\left\{\varphi_{\tau}\right\}$ where $\varphi_{1}=1$ and $\varphi_{\sigma}=\psi$. Let $f_{2}: G_{u} \rightarrow G$ be an isomorphism defined over $C$ such that $f_{2}^{\sigma} \circ f_{2}^{-1}=\psi$ and let $T_{u}=f_{2}^{-1}(T)$.

Let $G_{1}$ be a simply-connected absolutely simple algebraic group defined over $\boldsymbol{R}$ which is isomorphic to $G$ (and, hence, $G_{u}$ ) over $\boldsymbol{C}$. The isomorphism $f_{1}: G_{1} \rightarrow G_{u}$ may be chosen so that $f_{1}^{\sigma} \circ f_{1}^{-1}=\theta_{u} \circ I_{t_{u}}$ where $t_{u}$ is an $\boldsymbol{R}$-rational element of $T_{u}$, $t_{u}^{2} \in Z\left(G_{u}\right)$, and $f_{2} \theta_{u} f_{2}^{-1}=\theta \in \Theta[4$, Théorème 1, p. 295 and Théorème 2, p. 303]. We put $f=f_{2} \circ f_{1}$. Then $f^{\sigma} \circ f^{-1}=\psi \circ \theta \circ I_{t}$ where $t=f_{2}\left(t_{u}\right)$. Since $t_{u}^{2} \in Z\left(G_{u}\right)$, it follows that $t^{\sigma}=f_{2}^{\sigma}\left(t_{u}\right)=\psi f_{2}\left(t_{u}\right)=f_{2}\left(t_{u}^{-1}\right)=t z$ where $z \in Z(G)$. Furthermore, putting $\alpha=\theta \circ I_{t}$, we see that $\alpha^{\sigma}=\alpha$.

Finally, we should remark that explicit expressions for the elements $t_{u}$ are given in [4].

\section{BIBLIOGRAPHY}

1. F. Grosshans, Orthogonal representations of algebraic groups, Trans. Amer. Math. Soc. 137 (1969), 519-531.

2. A. I. Mal'cev, On semisimple subgroups of Lie groups, Izv. Akad. Nauk SSSR Ser. Mat. 8 (1944), 143-174; English transl., Amer. Math. Soc. Transl. (1) 9 (1962), 172-213. MR 6, 146.

3. G. D. Mostow, Some new decomposition theorems for semi-simple groups, Mem. Amer. Math. Soc. No. 14 (1955), 31-54. MR 16, 1087.

4. S. Murakami, Sur la classification des algèbres de Lie réeles et simples, Osaka J. Math. 2 (1965), 291-307. MR 34 \#1460.

5. I. Satake, Symplectic representations of algebraic groups satisfying a certain analyticity condition, Acta Math. 117 (1967), 215-279. MR 35 \#6694.

6. T. Tsukamoto, On the local theory of quaternionic antihermitian forms, J. Math. Soc. Japan 13 (1961), 387-400. MR 25 \#127.

JOHNS HOPKINS UNIVERSITY,

Baltimore, Maryland 21218 